\title{
Ortorexia nervosa e exercício físico: revisão de literatura
}

\author{
Orthorexia nervous and exercise: literature review
}

Resumo Recentemente, a procura por um corpo visto como "ideal" tem gerado uma constante preocupação com os aspectos nutricionais. $\mathrm{O}$ comportamento obsessivo por uma dieta dita como saudável pode trazer riscos para a saúde do indivíduo, tanto no âmbito biológico quanto social. Esse padrão nutricional compulsivo tem sido denominado ortorexia nervosa, cujas características e implicações ainda são debatidas. Especula-se se há alguma relação entre a prática regular de exercício físico e o desenvolvimento de um comportamento ortoréxico. O conceito é recente, porém, as implicações desse comportamento em relação à saúde e qualidade de vida apontam para a importância de estudos que conduzam à compreensão do quadro clínico. Nesse sentido, o presente trabalho tem por objetivo pesquisar os estudos já existentes sobre a ortorexia nervosa e as suas relações com o exercício físico. Utilizando as palavras-chave ortorexia e exercício e seus correspondentes em inglês, foram acessados 16 textos no google acadêmico e PubMed. Há consenso que o comportamento alimentar obsessivo pode trazer variáveis consequências físicas, psíquicas e sociais ao indivíduo. Alguns estudos relacionando ortorexia e exercício, discutem a associação dos comportamentos a objetivos de perda de peso e insatisfação com a imagem corporal e outros discutem esses quadros associados a necessidades atléticas e competitivas. Entretanto, por ser um conceito recente, os estudos a respeito da ortorexia nervosa ainda são escassos e mostram aspectos e conclusões diferentes. Por não haver um consenso entre os pesquisadores sobre a etiologia, os critérios diagnósticos, a sintomatologia e as formas de tratamento desse distúrbio, novas pesquisas são necessárias. Esta revisão aponta, além da carência de estudos na área, uma limitação das ferramentas de diagnóstico existentes para a identificação da ortorexia nervosa, bem como, das suas possíveis relações com a prática de exercícios físicos.

Palavras-chave: Ortorexia. Exercício.

Silvia Cristina Crepaldi Alves ${ }^{\mathrm{I}}$ FÁbio Gardin Pellegrini ${ }^{1}$ LYDIA IGNEZ ROMERA ${ }^{\mathrm{I}}$

${ }^{1}$ Universidade Metodista de Piracicaba (UNIMEP). Piracicaba/SP - Brasil.
Abstract Recently, the hunt for a said "ideal" body shape resulted in a constant concern about nutrition. Obsessive behavior in search for a diet aforementioned healthy can bring dangers to an individual in one's social and biological aspects. This compulsive obsessive eating pattern has been coined as "Orthorexia Nervosa", which carachteristics and entanglement are yet to be determined. It is still hypothesized if regular exercise practice has any role in orthorexic behavior development. Furthermore, the concept is recent therefore literature regarding the theme is scarce. Hence new studies that lead to the comprehension of the clinical picture shall be conducted. The aim of this paper is to research present 
studies related to orthorexia nervosa and its relationships with exercise. Using key words ortorexia and exercício, and its English analogous words sixteen papers were found throught Google Scholar a PubMed. There is consensus that obsessive eating patterns can lead to physical, psychic and social consequences to the individual. Some studies connecting orthorexia nervosa and exercise argue about the relationship between obsessive behavior's and weight loss, and body image while other studies discuss athletical needs and competition. Nevertheless, it was identified that due to the fact the term has been coined recently studies about "orthorexia nervosa" are meager and its aspects and conclusions may differ. By not reaching a consensus among researchers, etiology, diagnostics criteria, symptomatology and treatment of this disorder still have a long way to go still needing further discussion and studies. This paper shows beyond the lack of studies in the aforementioned area and the current limitations of the existent diagnostic instruments employed to identify "orthorexia nervosa", as well, its possible relationship with exercise.

KEYWORDS: ORTHOREXIA. EXERCISE.

\section{INTRODUÇÃO}

Atualmente, o bem-estar, a alimentação, o exercício e a imagem corporal são conceitos, fortemente associados aos cuidados com a saúde, ${ }^{1}$ mas, a exacerbação desse comportamento e as preocupações com a saúde também podem ser consideradas patológicas. ${ }^{2}$ Nesse mesmo contexto, estão inseridos os conceitos de distúrbios alimentares já reconhecidos, como anorexia, bulimia e vigorexia., ${ }^{1,2}$

Muitos autores têm discutido a gênese e características dos transtornos alimentares e dos padrões emergentes de comportamentos alimentares relacionados com a prática de exercícios e o zelo excessivo pela imagem corporal; nessas discussões, surge o conceito de comportamento ortoréxico. ${ }^{2}$

A ortorexia tem sido conceituada, de forma geral, como a fixação pelo consumo de alimentos saudáveis, caracterizada pela demasiada preocupação com a alimentação, tornando a seleção de alimentos uma obsessão, podendo trazer consequências negativas para o indivíduo. Apesar da atenção à alimentação saudável ser importante, a exa- cerbação desse comportamento torna-se um distúrbio alimentar, ganhando interesse público devido aos efeitos severos que a mesma pode trazer nos âmbitos psíquicos, sociais, laborais, acadêmicos e na qualidade de vida. ${ }^{2}$

Existem grupos mais vulneráveis para o desenvolvimento da ortorexia nervosa; com destaque aos praticantes de exercício físico e frequentadores de academia, mas ainda não são claras as inter-relações entre esses comportamentos. Há dúvidas, por exemplo, se apenas participantes de atividades fitness estão em um grupo de risco, ou se atletas de alto rendimento fazem parte desse grupo. ${ }^{2}$

A ortorexia nervosa ainda não é considerada oficialmente um transtorno alimentar devido à sua não inclusão no DSM-5 (Manual de estatística e diagnóstico dos transtornos mentais - 5 edição) assim como, no CID-10 (Código Internacional de Doenças), nem mencionados na sessão específica de transtornos que requerem mais estudos. Porém, seu conceito, suas características, interações, sintomas e etiologia ainda são discutidos. ${ }^{2}$

Portanto, estudos que conceituem e discutam a ortorexia e suas relações com o 
exercício físico são relevantes à compreensão e orientação de condutas profissionais. Nesse sentido, o objetivo deste estudo é realizar uma revisão de literatura acerca do tema ortorexia de forma específica, e a sua relação com a prática de exercício físico.

\section{Métodos}

As pesquisas foram feitas sem definição de limite de tempo das publicações, com base no indexador Google Acadêmico, utilizando as palavras-chave "ortorexia" e "exercício" e no PubMed, usando os correspondentes em inglês, "orthorexia" e "exercise".

Excluindo-se patentes, citações e filtrando os resultados para textos redigidos em português e de livre acesso, retornaram 60 resultados. Após triagem inicial, exclusão de duplicatas, e artigos que não atendiam aos critérios de relevância, restaram 30 artigos.

A pesquisa no PubMed para a palavra-chave "orthorexia" retornou 67 textos, considerando-se apenas artigos de livre acesso, a pesquisa reduziu para 16 publicações. Quando foi associada a palavra-chave "exercise" ao mecanismo de busca, a pesquisa retornou apenas dois artigos.

Para a redação da presente revisão foram analisados 16 artigos em português e inglês, e cujos conteúdos eram essencialmente pertinentes à temática "ortorexia".

\section{Desenvolvimento}

\section{CONCEITUALIZAÇÃO}

A ortorexia nervosa foi proposta primeiramente por Bratman, ${ }^{3}$ em 1997, que a definiu como a fixação por comer alimentos saudáveis.
O fato de ser um conceito recente torna a ortorexia nervosa um comportamento difícil de ser classificado, portanto, ainda não há uma definição científica precisa e consensual. Pesquisadores e profissionais da saúde discutem se a ortorexia deve ser considerada um transtorno alimentar, um vício comportamental ou um hábito dietético extremo. ${ }^{4}$

A literatura atual é ambígua e não fornece clareza suficiente para caracterizar a ortorexia nervosa como um distúrbio alimentar específico ou apenas uma vertente de algum transtorno alimentar preexistente. ${ }^{5}$

Martins et al. (2011) ${ }^{6}$ complementaram a caracterização feita por Bratman (1997), ${ }^{3}$ citando outros sintomas e propondo que a ortorexia é um distúrbio de personalidade ou de comportamento.

É importante salientar que, a ortorexia nervosa não é caracterizada como transtorno alimentar por não constar em qualquer manual específico sobre patologias. Entretanto, há consenso no fato de que o comportamento obsessivo por uma alimentação saudável ou uma dieta dita como saudável traz consequências físicas, psíquicas e sociais ao indivíduo. ${ }^{2}$

\section{Sintomatologia}

Bratman, em 1997, argumentou que a ortorexia tem seu início geralmente num ato inocente de controle alimentar para superar uma doença ou simplesmente para haver uma melhora da saúde de uma maneira geral. Porém, em certos casos de reeducação alimentar, principalmente naqueles em que deve haver uma mudança radical dos hábitos alimentares (quando comparados aos da infância ou da cultura do meio inserido, por exemplo), poucos indivíduos conseguem contemplar essa mudança sem maiores in- 
tercorrências. Muitas vezes recorrem a um regime de autodisciplina superinflado; e com o tempo, o que comem, o quanto comem, e as consequências dos alimentos que ingerem acabam por ocupar uma proporção demasiada em suas vidas, onde colocam sua dieta como prioridade absoluta, caracterizando assim o comportamento ortoréxico. O ortoréxico passa a ser dominado por esforços de resistir a tentações, autocondenação por lapsos, autopromoção por sucessos conseguidos no seu regime alimentar e uma sensação de superioridade frente àqueles que possuem uma dieta "menos pura".

A ortorexia nervosa foi diretamente relacionada a costumes religiosos, filosóficos e financeiros. Similaridades entre a ortorexia nervosa e outros transtornos alimentares relatando a ansiedade, necessidade de controle e seguimento de regras rígidas, desejo de se sentir puro e tendência ao perfeccionismo, tidos como as principais congruências entre os distúrbios de comportamento alimentar, foram apontados. Além disso, existem evidências que a maioria dos transtornos alimentares é motivada por pressões sociais. ${ }^{6}$

Estudos apontam que a ortorexia nervosa compartilha sintomas relacionados a patologias alimentares obsessivas compulsivas já conhecidas, como a anorexia e bulimia. Tais sintomas se apresentam como foco excessivo em tópicos relacionados a alimentos, dieta restritiva, perfeccionismo, co-ocorrência de ansiedade, necessidade de controle, rigidez comportamental e até mesmo "rituais" relacionados à preparação da refeição. Mas salientam que, no indivíduo ortoréxico, características marcantes da anorexia e bulimia, como preocupação excessiva com a perda de peso, medo de ganhar peso e superestimação do tamanho corporal podem estar ausentes. ${ }^{5}$
Estudos complementam que o indivíduo ortoréxico tem crenças distorcidas em relação a hábitos alimentares, o que os leva a dar importância demasiada à percepção de salubridade dos alimentos e seus valores nutricionais, em detrimento à apreciação do alimento e ao seu sabor. Em alguns casos, há o foco específico em ingerir apenas alimentos livres de laticínios, veganos, vegetarianos ou alimentos em seu estado natural (sem cozimento). A origem, qualidade, embalagem e processamento dos alimentos são escrutinizados devido à preocupação crescente em ingerir apenas alimentos considerados saudáveis. Além da preocupação com os alimentos em si, uma grande porção do tempo do ortoréxico é consumida por pensamentos deletérios sobre sua alimentação e preocupação crônica quanto a possíveis falhas em sua dieta. ${ }^{7}$

\section{POSSÍVEIS CONSEQUENNCIAS DA ORTOREXIA NERVOSA}

Os estudos de Dell Osso et al., ${ }^{7}$ em 2016, discutem a existência de uma teórica coincidência entre os sintomas da ortorexia e da anorexia, ambas consideradas pelos autores como transtornos obsessivos compulsivos. Portanto, é de se esperar que assim como há a sobreposição de sintomas, há também a sobreposição de sequelas. O indivíduo com ortorexia tem um conhecimento pobre sobre as consequências de seu comportamento e os riscos em âmbitos profissionais, sociais e de aprendizado. A demasiada preocupação e a ênfase excessiva no que o indivíduo considera como alimentação saudável acaba por tirar o foco de assuntos importantes, como o relacionamento interpessoal, o trabalho e os estudos. ${ }^{7}$

Pesquisadores afirmam que o comportamento alimentar obsessivo traz con- 
sequências físicas, psíquicas e sociais ao indivíduo. No âmbito social, apontam que há um distanciamento por parte dos ortoréxicos, geralmente devido ao fato de se acharem na obrigação de esclarecer a todos sobre a importância com o cuidado alimentar, isso resulta no desenvolvimento de um sentimento de desprezo perante os que, na sua visão, não têm hábitos saudáveis de alimentação. ${ }^{2} \mathrm{O}$ planejamento alimentar estrito também pode afetar a frequência do ortoréxico em eventos sociais como churrascos, restaurantes, e eventos familiares; diminuindo ou até cessando o seu comparecimento, devido ao fato de não "confiarem" no alimento servido. Como consequência psíquica destacam que a obsessão por um corpo saudável, leva à adoção de comportamentos dietéticos distorcidos e exagerados. Portanto, o comportamento ortoréxico que visa à saúde, traz à pessoa exatamente o contrário. ${ }^{2}$

Em um relato de caso, pesquisadores avaliaram um indivíduo com ortorexia nervosa severa que desenvolveu sérias patologias devido à sua dieta extremamente restritiva; em sua maioria relacionadas à deficiência de elementos essenciais para a manutenção da saúde. Concluíram que a ortorexia nervosa pode ter manifestações variáveis podendo estas serem até fatais. ${ }^{8}$

\section{PREVALÊNCIA E MÉTODOS DE DIAGNÓSTICO}

As pesquisas relacionadas à prevalência do comportamento ortoréxico, na população mundial, apresentam resultados muito variáveis. Existem dados contraditórios em relação à comparação da incidência ser maior em homens ou em mulheres. ${ }^{9}$ Por sua vez, vale salientar, estudos que propõem que a falta de ferramentas para diagnóstico, a falta de consenso entre medidas de avaliação psicométricas adotadas, e mesmo a não caracterização como patologia, fazem que qualquer estimativa de prevalência não seja digna de credibilidade. ${ }^{10}$

Para o diagnóstico de ortorexia, foi proposto inicialmente um questionário chamado de "Orthorexia Self Test", o qual compreende dez questões que são aplicadas ao indivíduo. Suas respostas se baseiam numa escala dicotômica SIM/NÃO e, ao final do teste, a pessoa é considerada ortoréxica caso tenha resposta positiva para quatro ou mais questões. Porém, esse teste não é validado, assim não podendo ser usado como ferramenta confiável para a avaliação. ${ }^{11}$

Um instrumento adequado para a avaliação de comportamento ortoréxico é o teste ORTO-15, questionário desenvolvido pelo pesquisador italiano Donini e colaboradores, em 2005, que compreende 15 questões avaliadas numa escala Likert de quatro pontos. Esse questionário analisa as inter-relações entre aspectos cognitivos-racionais, clínicos e emocionais. O ORTO-15 avalia conceitos sobre as atitudes perante a seleção de alimentos, até que ponto a alimentação influencia a vida cotidiana, os efeitos percebidos da alimentação saudável e hábitos de consumo de alimentos. Valores baixos nos escores revelam maior tendência à ortorexia nervosa, ou seja, quanto menor a pontuação no questionário tanto maior risco à ortorexia nervosa aquele indivíduo apresentará. ${ }^{12}$

Os pesquisadores brasileiros Pontes et al. ${ }^{13}$ desenvolveram a tradução e adaptação cultural do questionário italiano ORTO-15 para o português, como pode ser observado no Quadro 1. No mesmo quadro, estão especificados os valores numéricos correspondentes às respostas, segundo Missbach et al. ${ }^{12}$ 
Quadro 1: Questionário ORTO-15, usado como ferramenta para diagnóstico de ortorexia nervosa, contendo 15 questões e quatro opções de respostas para cada uma delas com pontuações específicas. ${ }^{12,13}$

\begin{tabular}{|c|c|c|c|c|}
\hline $\begin{array}{l}\text { Marcar com um X a alternativa que melhor } \\
\text { corresponde ao seu comportamento em relação } \\
\text { à comida }\end{array}$ & SEMPRE & $\begin{array}{l}\text { MUITAS } \\
\text { VEZES }\end{array}$ & $\begin{array}{l}\text { ALGUMAS } \\
\text { VEZES }\end{array}$ & NUNCA \\
\hline $\begin{array}{l}\text { 1. Você fica atento(a) às calorias dos alimentos } \\
\text { quando come? }\end{array}$ & 2 & 4 & 3 & 1 \\
\hline $\begin{array}{l}\text { 2. Quando você vai a um mercado de alimentos, } \\
\text { se sente confuso a respeito do que deve comprar? }\end{array}$ & 4 & 3 & 2 & 1 \\
\hline $\begin{array}{l}\text { 3. Nos últimos três meses, pensar sobre sua } \\
\text { alimentação tem sido uma preocupação? }\end{array}$ & 1 & 2 & 3 & 4 \\
\hline $\begin{array}{l}\text { 4. As suas escolhas alimentares são determinadas } \\
\text { pela preocupação com seu estado de saúde? }\end{array}$ & 1 & 2 & 3 & 4 \\
\hline $\begin{array}{l}\text { 5. O sabor é a qualidade mais importante que } \\
\text { você leva em consideração, ao escolher um } \\
\text { alimento? }\end{array}$ & 4 & 3 & 2 & 1 \\
\hline $\begin{array}{l}\text { 6. Normalmente, você se dispõe a pagar mais por } \\
\text { alimentos saudáveis? }\end{array}$ & 1 & 2 & 3 & 4 \\
\hline $\begin{array}{l}\text { 7. A preocupação com alimentação saudável } \\
\text { toma mais de três horas do seu dia? }\end{array}$ & 1 & 2 & 3 & 4 \\
\hline $\begin{array}{l}\text { 8. Você se permite alguma quebra da sua rotina } \\
\text { alimentar? }\end{array}$ & 4 & 3 & 2 & 1 \\
\hline $\begin{array}{l}\text { 9. Para você, o seu humor influencia o seu } \\
\text { comportamento alimentar? }\end{array}$ & 4 & 3 & 2 & 1 \\
\hline $\begin{array}{l}\text { 10. Você acredita que a convicção de se alimentar } \\
\text { saudavelmente aumenta sua autoestima? }\end{array}$ & 1 & 2 & 3 & 4 \\
\hline $\begin{array}{l}\text { 11. Você acha que o consumo de alimentos } \\
\text { saudáveis modifica seu estilo de vida (ida a } \\
\text { restaurantes, amigos...)? }\end{array}$ & 1 & 2 & 3 & 4 \\
\hline $\begin{array}{l}\text { 12. Você acredita que consumir alimentos } \\
\text { saudáveis pode melhorar o seu aspecto físico? }\end{array}$ & 1 & 2 & 3 & 4 \\
\hline $\begin{array}{l}\text { 13. Sente-se culpado(a) quando sai da sua rotina } \\
\text { alimentar? }\end{array}$ & 2 & 4 & 3 & 1 \\
\hline $\begin{array}{l}\text { 14. Você pensa que no mercado existem } \\
\text { alimentos não saudáveis? }\end{array}$ & 1 & 2 & 3 & 4 \\
\hline $\begin{array}{l}\text { 15. Ultimamente, você costuma estar sozinho(a) } \\
\text { quando se alimenta? }\end{array}$ & 1 & 2 & 3 & 4 \\
\hline
\end{tabular}


É importante salientar que Håman et al. $(2015)^{4}$ pontuam em seu estudo que o questionário ORTO-15 não é totalmente preciso devido à limitações e sensibilidade desse instrumento. Nesse questionário, não são contempladas questões referentes a comportamentos culturais, por exemplo, também não há uma análise longitudinal dos fatores abordados, além disso, não existem adequações necessárias para a avaliação pertinente de variações temporais e ambientais. Não obstante, o questionário tem sido o mais utilizado como ferramenta para o diagnóstico da ortorexia nervosa.

\section{ORTOREXIA NERVOSA E EXERCÍCIO FÍSICO}

Foi revelada uma relação entre esportes e exercícios e escores baixos no ORTO-15, o que pode revelar uma maior prevalência desse comportamento em praticantes assíduos de exercícios físicos. Porém, resultados positivos no teste ORTO-15 para ortorexia não significam necessariamente que aquele indivíduo é ortoréxico. Devido a fatores culturais e sociais, certos nichos populacionais tendem a ter um comportamento alimentar regrado. Por exemplo, praticantes de yoga ashtanga, em sua grande maioria, respondem positivo para a ortorexia, mas esse tipo de atividade requer em seu cerne autodisciplina e modificações do estilo de vida, como alimentação saudável, portanto, esse grupo não pode ser considerado ortoréxico, sendo o seu comportamento apenas um reflexo do seu estilo de vida. No entanto, devemos considerar que a detecção de uma alta tendência para ortorexia, por meio da aplicação do ORTO-15, não representa necessariamente um comportamento patológico. ${ }^{4}$
Num estudo onde foram avaliadas diferentes respostas para indivíduos com distúrbios alimentares, foram analisadas 52 mulheres diagnosticadas com distúrbios alimentares (ortorexia, anorexia e bulimia), e se procurou identificar diferentes preditores para ortorexia ao relacioná-la com anorexia e bulimia. Os resultados pontuam que $44,2 \%$ (23 mulheres) relacionavam o exercício físico como maneira para perder peso de maneira intencional, logo podendo ter alguma relação com a ortorexia. De acordo com uma equação proposta pelos pesquisadores deste estudo, a ortorexia é resultado de uma associação de fatores, entre eles a avaliação pessoal da condição física e atitude frente a exercícios físicos. Contudo, os autores não conseguiram fazer uma associação explícita entre a prática de exercícios físicos e a ortorexia. ${ }^{14}$

Existe uma influência direta da cultura, aspectos profissionais, e de lazer, nos resultados de escores baixos ao questionário ORTO-15, o que poderia indicar a presença de ortorexia. Esses achados foram evidentes entre grupos de artistas de performance (cantores de ópera, dançarinos de ballet e músicos sinfônicos), porém, os resultados altos podem ser reflexo de um nível socioeconômico e cultural elevado, e, adicionalmente, artistas são um modelo para o público em geral em termos de aparência e estilo de vida, portanto, escores altos na avaliação não significam necessariamente que o indivíduo tem ortorexia. ${ }^{4}$

Martins et al. (2011) discutem que adolescentes esportistas do sexo feminino dedicadas ao fisiculturismo e atletismo poderiam ser mais vulneráveis à ortorexia. Em seu trabalho, associam a ortorexia nervosa ao exercício físico diretamente, observando 
que mulheres praticantes de exercício físico frequente e com objetivo de obter forma corporal mais aceita pela sociedade contemporânea apresentaram escores elevados para ortorexia em ferramentas de avaliação. Sendo assim, eles propõem que crenças e comportamentos ortoréxicos podem também estar relacionados à busca do corpo perfeito, o qual tem relação direta com o exercício físico. ${ }^{6}$

Seguindo essa linha de raciocínio, Ainett et al. (2017) $)^{15}$ investigaram a prevalência de insatisfação com a imagem corporal e fatores associados em estudantes de nutrição. Apontam que a insatisfação com a imagem corporal do indivíduo vem à tona quando o ato de comparar o seu corpo com o de outras pessoas gera um sentimento de negação, e dependendo do seu grau, pode afetar aspectos de vida do indivíduo, principalmente no que se refere aos seus hábitos alimentares. O fato de haver uma difusão massiva de modelos corporais magros e musculosos, como sendo sinônimo de beleza e atratividade, coopera com o aumento de indivíduos que não se enquadram nesses padrões e têm uma grande propensão a desenvolver uma percepção negativa da imagem corporal, mesmo estando num estado eutrófico. Essa insatisfação pode levar a comportamentos alimentares impróprios, que podem se desenvolver para transtornos alimentares, entre eles a ortorexia. Em suas pesquisas, chegaram à conclusão que $69,2 \%$ da amostra estudada (133 indivíduos, 80,5\% mulheres, $19,5 \%$ homens) apresentaram insatisfação com a imagem corporal.

Um estudo proposto em 2009 analisou a prevalência de ortorexia nervosa em estudantes jovens do sexo feminino de cursos da área de saúde, supondo uma maior vulne- rabilidade desse grupo à ortorexia nervosa. Contudo, concluíram não haver indícios de comportamento ortoréxico, sugerindo que esse quadro seja específico de populações já adeptas a algum tipo de restrição alimentar devido à escolhas prévias. ${ }^{16}$

Visto que há associação com a insatisfação com a imagem corporal e transtornos alimentares, deve haver uma preocupação entre a associação desses fatores. ${ }^{15,16}$

Atletas podem estar num grupo de risco elevado para o desenvolvimento da ortorexia devido ao fato que esses indivíduos são particularmente sensíveis a fatores relacionados à saúde e nutrição e também podem em determinado ponto sentir certa pressão para serem bons exemplos no tocante à saúde, desse modo desenvolvendo tendências ao perfeccionismo alimentar. Entretanto, essa relação não é clara, sendo assim é importante examinar de maneira longitudinal e atenciosa esse grupo. ${ }^{10}$

Em contrapartida, Håman et al. $(2015)^{4}$ consideram que o comportamento de restrição alimentar faz parte da "identidade" de um atleta, principalmente aqueles envolvidos em artes marciais, e atletas de elite, mas essa tendência pode estar intimamente relacionada com a cultura desportista. Em seu estudo propõem que hábitos alimentares rígidos podem ser considerados como práticas normais entre atletas de performance, enquanto os mesmos hábitos são considerados incorretos por indivíduos ativos. Ainda, denotam que atletas que obtiveram escores altos para ortorexia são fruto da necessidade de controle alimentar para a sua performance atlética, e que esses indivíduos não podem ser considerados ortoréxicos por não apresentarem esse comportamento 
de maneira contínua. Essa disparidade demonstra a importância de se contextualizar o comportamento ortoréxico.

\section{CONSIDERAÇões FINAIS}

Ortorexia nervosa é um termo descrito recentemente, portanto estudos referentes a esse assunto ainda são exíguos. $\mathrm{O}$ fato de não haver uma definição clara desse comportamento como patologia dificulta a identificação de sua prevalência e, por consequência, pesquisas para a ampliação do conhecimento acerca do tema. Os meios para o diagnóstico, por sua vez, não contemplam todas as variáveis necessárias para a identificação do quadro ortoréxico, em diferentes nichos populacionais, tornando a constatação da presença do distúrbio bastante complexa. Considerando que a ortorexia nervosa é multifatorial, ou seja, seu desenvolvimento é dependen- te de diversos fatores isolados, que juntos resultam no distúrbio, identificar a relação dessas variáveis é excepcionalmente intricada. A literatura ainda é dicotômica sobre a relação dos exercícios físicos e esportes no desenvolvimento da ortorexia. Por conseguinte, novos estudos devem ser conduzidos com o propósito de identificar essa possível relação e suas variáveis.

Embora existam, atualmente, ferramentas que podem ser usadas como indicativas da presença de ortorexia nervosa, as mesmas não estabelecem relações com a prática de exercícios. Especificamente, o questionário ORTO-15 parece generalista e carece de acurácia no tocante à variações temporais, sociais e comportamentais dos indivíduos avaliados. Um maior detalhamento desses itens possivelmente geraria resultados mais elucidativos e, por conseguinte, uma maior capacidade para a compreensão da ortorexia nervosa e das suas relações com a prática de exercícios.

\section{REFERÊNCIAS}

1 Baptista TJR. A obesidade e indústria do emagrecimento. ComCiência, fev. 2013; 145.

2 Coelho GC, Troglio GM Hammes L. Galvão TD, Cyrino LAR. As consequências físicas, psíquicas e sociais em indivíduos com ortorexia nervosa. Revista Brasileira de Obesidade, Nutrição e Emagrecimento, mai./jun. 2016; 10 (57): 160-168.

3 Bratman. Original essay on orthorexia, 1997 [acesso 9 mar. 2017]. Disponível em: <://www. orthorexia.com/original-orthorexia-essay/>

4 Haman L., Rughti NB, Patriksson G., Lindgren EC. Orthorexia nervous: An integrative literature review of a lifestyle syndrome Qualitative Studies on Health and Well-being, 2015; 10 (1): 267-299.

5 Gramaglia C., Matera AB, Rogoza R., Zeppegno P. Orthorexia and anorexia nervosa: two distinct phenomena? A cross-cultural comparison of orthorexic behaviours in clinical an non-clinical samples. BMC Psychiatry, 2017; 17 (1): 75-80.

6 Martins MCT, Alvarenga MS, VArgas SVA, Sato KSCJ, Scagliusi FB. Ortorexia nervosa: Reflexões sobre um novo conceito. Revista de Nutrição, Campinas, mar./abr. 2011; 24 (2): 345-357.

7 Del Osso et al. Historical evolution of the concept of anorexia nervosa and relationships with orthorexia nervosa, autism, and obsessive-compulsive spectrum. Neuropyschiatric Disease and Treatment, 2016; 12: 1.651-1.660. 
8 Park SW, et al. Orthorexia nervous with Hyponatremia, Subcutaneous Emphysema, Pneumomediastimum, Pneumothorax and Pancytopenia. The Korean Society of Electrolyte Metabolism, 2011; 9: 32-37.

9 Michalska, A., et al. Nonspecific eating disorders-a subjective review. Psychiatria polska, 2016; 50 (3): 497-507.

10 Koven NS, Abry AW. The clinical basis of orthorexia nervosa: emerging perspectives. Neuropyschiatric Disease and Treatment, 2015; 11: 385-394.

11 Stotchel et al. Validation of ORTO-15 Questionnaire in the group of urban youth aged 15-21. Psychiatr. Pol., 2015; 49 (1): 119-134.

12 Misbach B., Hinterbuchinger B., Dreseiti V., Zellhofer S., Kurz C., König J. When eating right, is measured wrong! A validation and critical examination of the ORTO-15 questionnaire in German 2015; 10 (8) 1-15.

13 Pontes JB, Montagner MI, Montagner MA. Ortorexia Nervosa: adaptação cultural do ORTO-15. Alimentação Nutrição e Saúde, 2014; 9 (2): 593-548.

14 Matera AB, Rogoza R., Gramaglia C., Zeppegno P. Predictors of orthorexic behaviours in patients with eating disorders: a preliminary study. BMC Psychiatry 2015; 15: 252-260.

15 Ainett WSO, Costa, VVL, Sá, NNB. Fatores associados a insatisfação com a imagem corporal em estudantes de nutrição. Rev. Bras. Obesidade, Nutr. e Emagrecimento, 2017; 11 (62): 75-78.

16 Lopes, MR; Kirsten, VR. Comportamentos de ortorexia nervosa em mulheres jovens. Disciplinarum Scientia Saúde, 2016; 10 (1): 97-105.

\section{DAdos DOS AUTORES}

\section{Silvia Cristina Crepaldi Alves}

Graduada em Ciências Biológicas pela Universidade Estadual de Londrina. Mestrado e Doutorado em Fisiologia e Biofísica pela Universidade Estadual de Campinas (UNICAMP). Docente na Universidade Metodista de Piracicaba (UNIMEP).Piracicaba/SP - Brasil. silvia.alves@unimep.br

\section{Fábio Gardin Pellegrini}

Graduado em Educação Física pela Universidade Metodista de Piracicaba (UNIMEP). Graduado em Odontologia pela Universidade de Ribeirão Preto (UNAERP). Mestrando em Ciências da Nutrição e do Esporte e Metabolismo na Universidade Estadual de Campinas (UNICAMP). Limeira/SP - Brasil. pellegrinimtb@hotmail.com

\section{LYDIA IGNEZ ROMERA}

Graduada em Educação Física pela Universidade Metodista de Piracicaba (UNIMEP). Coordenadora técnica de Academia. Americana/SP - Brasil. lydiaromera@yahoo.com.br

Submetido em: 22-11-2017

Aceito em: 6-1-2018 